\title{
Molecular theory of dendritic liquid crystals: self-organisation and phase transitions $\dagger$
}

\author{
Alexandros G. Vanakaras* and Demetri J. Photinos \\ Received 11th November 2004, Accepted 22nd March 2005 \\ First published as an Advance Article on the web 14th April 2005 \\ DOI: 10.1039/b416710d
}

We present tractable molecular theory descriptions of liquid crystalline dendrimers based on clearly defined approximations and in terms of the dominant interactions underlying the self-organisation of these large and complex supermolecular entities. We formulate the configurational partition function for dendrimers, taking explicit account of their conformations and segmental interactions. Two approximate schemes are presented: the first is based on the effective interactions of the dendrimers as a whole while the second scheme is based on the interactions among the mesogenic units contained in the dendrimers. Results of lattice calculations for phase transitions in the context of the first scheme are presented and they show that the minimal inclusion of shape anisotropy and of sub-molecular partitioning into chemically distinct parts is sufficient to reproduce the variety of phases and phase sequences observed experimentally and provides insights into the conformational aspect of these transitions. In the second scheme, the description of the dendromesogenic system reduces to that of an ensemble of mesogenic dimers. This scheme can be readily extended to the description liquid crystalline oligomers and polymers consisting of mesogenic units connected by flexible spacers in various architectures. It thus provides a unified approach for treating mesomorphic phase transitions of supermolecular and macromolecular systems that can be built by connecting the same submolecular units in topologically different ways.

\section{Introduction}

The study of liquid crystalline phases formed by various types of dendritic systems ${ }^{1-13}$ differing in their architecture, in the chemical structure of the branches, the functionalisation of the surface $e t c$., has led to the identification of several possible mechanisms of supermolecular self organisation. ${ }^{14}$ These include:

(a) Micro-segregation, generated by the partitioning of the dendritic structure into chemically distinct regions. This mechanism is believed to underlie the liquid crystalline selforganisation of dendrimers whose overall shape and the shapes of their subunits lack orientability. ${ }^{1}$

(b) Mutual alignment of mesogenic units. This mechanism is dominant in radial or globular dendrimers containing mesogenic units. The latter could be attached to the external periphery of the dendritic scaffold ${ }^{2-9}$ or be part of the scaffold as well. ${ }^{10}$ The orientational order results primarily from the anisotropic interactions among mesogenic units belonging to different dendrimers (inter-dendritic) or to the same dendrimer (intra-dendritic). The intra-dendritic interactions could induce an asymmetry to the overall shape of the dendrimer that, in turn, enhances the orientational order. On the other hand, in systems with a flexible non-mesogenic dendritic scaffold and peripherally attached mesogenic units,

$\uparrow$ This work was funded as part of the EU Research Training Network "LCDD”, Supermolecular Liquid Crystal Dendrimers.

*vanakara@upatras.gr the micro-segregation mechanism, stemming from the chemical distinction between the flexible scaffold and the mesogenic periphery, is superimposed to the mesogenic interactions and promotes partial positional order in the form of layering or column formation. ${ }^{2-9}$

(c) Self-assembly of dendritic units to form supramolecular structures that self-organise into liquid crystalline phases. ${ }^{11}$ The structure and interactions of the dendra, which need not contain any intrinsically mesogenic segments, control the shape of the supramolecular entities and thereby the symmetry of the ordered phases.

(d) Direct self-organisation of relatively rigid supermolecular structures, such as worm-like polymers or rigid rods ${ }^{12,13}$ formed by the bonding of dendritic units. The shape of the superstructure is determined by the way in which the dendritic units are bonded while the overall rigidity can be controlled by the generation of the dendra.

A basic issue in formulating any molecular theory of dendritic mesomorphism is the extent of detail to which the structure, the conformations and the interactions of the dendritic units are to be described. The molecular size of these systems and the usually enormous number of conformational states they can access precludes a fully atomistic description and one has therefore to identify and retain only the elements that are of primary relevance to their mesomorphic behaviour. The complexity of the problem stems from the presence of a broad hierarchy of interactions: dendrimers of a given generation have several topologically and chemically different segments and this gives rise to 
many combinations of intra- and inter-dendrimer segmental interactions. Moreover, the flexibility of these supermolecular structures is usually so extensive that even the treatment of the conformation statistics of a single dendrimer becomes extremely complex. ${ }^{15-21}$ Here we consider two simplified views of this problem. One view is to assign distinct roles to intra- and inter-dendrimer interactions by treating the dendrimers as deformable objects ${ }^{22-24}$ that can exist in a manageable number of conformational states predetermined by the intra-dendrimer interactions. These objects are then assumed to interact with each other as a whole in a way that is dictated entirely by the inter-dendrimer segmental interactions. The other view is to consider directly the interactions among the dendritic segments in a pair-wise manner (i.e. ignoring 3-segment correlations or higher) and impose on the intra-dendritic pairs the configurational constraints dictated by their connectivity within the same dendrimer. In other words, this "segmental" approach replaces the dendritic connectivity by a set of pair-wise configurational constraints on the segments of the same dendrimer.

The purpose of this work is not to reach a quantitative description of dendritic liquid crystals starting from their detailed molecular structure but rather to seek insights into the experimental trends exhibited by these systems in terms of their dominant molecular characteristics. The two approaches to be presented here are based on different assumptions and approximations and they are mutually supplementing. Their applicability extends to dendromesogenic systems described under mechanisms (a) and (b) in the beginning of this section. The self-assembly mechanism described under (c) is not considered in this work neither is mechanism (d), which can be treated, at some level of coarse graining, by the molecular theory methods developed for rigid rod or semirigid worm-like polymers. ${ }^{25}$

The paper is organized as follows. The exact statistical mechanics of the dendrimer ensemble is formulated in section II and serve as the basis for specifying the subsequent approximations entailed by each of the two approaches. The interconverting shape approach is developed in section III and is illustrated by applying it to a simple model system of dendrimers exhibiting interconversions between calamitic and discotic shapes. The segmental pair-interaction approach is presented in section IV and its application is illustrated for a model dendrimer consisting of a flexible non-mesogenic dendritic scaffold that is peripherally functionalised with mesogenic units. The last part of this section deals with the generalization of the segmental approach to linear liquid crystalline oligomers and polymers. Finally, the conclusions are presented in section $\mathrm{V}$.

\section{Formulation of the dendrimer ensemble free energy}

We consider an ensemble of $N_{\mathrm{D}}$ identical dendrimers occupying volume $V$ at temperature $T$. The individual dendrimers are labeled by the indices $I, J \ldots=1,2,3 \ldots N_{\mathrm{D}}$. We denote the position of the $I^{\text {th }}$ dendrimer by $\boldsymbol{R}_{I}$, its orientation by $\Omega_{I}$ and the set of variables specifying its conformational state by $v_{I}$. The energy of the dendrimer at that state is $E\left(v_{I}\right)$. Let the interaction between two such dendrimers be described by the pair potential $U_{I, J}=$ $U\left(\boldsymbol{R}_{I, J} ; \Omega_{I, J} ; v_{I}, v_{J}\right)$, with $\boldsymbol{R}_{I, J}, \Omega_{I, J}$ denoting respectively the position and orientation of dendrimer $J$ relative to $I$. The conformational energy $E\left(v_{I}\right)$ is understood to originate from the interactions among the segments that form dendrimer $I$. Labeling these segments by the index $i_{I}, j_{I} \ldots=1,2,3 \ldots N_{\mathrm{s}}$, where $N_{\mathrm{s}}$ is the total number of the segments into which the dendrimer is subdivided, and assuming that the intradendrimer interactions can be represented by a pair-wise superposition of potentials $u\left(i_{I}, j_{I}\right)$ among its constituent segments we have

$$
E\left(v_{I}\right)=\sum_{i_{I}, j_{I}} u\left(i_{I}, j_{I}\right)
$$

Similarly, the dendrimer-dendrimer interaction potential $U_{I, J}$ is written as a superposition of potentials $u\left(i_{I}, j_{J}\right)$ among all the inter-dendrimer pairs of segments $i_{I}, j_{J}$,

$$
U_{I, J}=\sum_{i_{I}, j_{J}} u\left(i_{I}, j_{J}\right)
$$

The equilibrium configurational partition function for this ensemble of dendrimers is then

$$
Q=\int \mathrm{d}\{I\} \prod_{I=1}^{N_{\mathrm{D}}} \mathrm{e}^{-E^{*}\left(v_{I}\right)} \prod_{J=I+1}^{N_{\mathrm{D}}} \mathrm{e}^{-U_{I, J}^{*}}
$$

with $\{I\}$ denoting collectively the complete set of configurational variables $\left\{\boldsymbol{R}_{I} ; \Omega_{I} ; v_{I}\right\}$ of the $N_{\mathrm{D}}$ dendrimer ensemble, $E^{*}\left(v_{I}\right) \equiv E\left(v_{I}\right) / k_{\mathrm{B}} T$ and $U_{I, J}^{*} \equiv U_{I, J} / k_{\mathrm{B}} T$.

The partition function of eqn. (3) can be put in an alternative form such that the purely conformational singledendrimer contribution, denoted by $q_{\mathrm{D}}$, is factored out of the part associated with the inter-dendrimer interactions and denoted by $Q^{\prime}$, i.e.

$$
Q=\left(q_{\mathrm{D}}\right)^{N_{\mathrm{D}}} Q^{\prime}
$$

where the single dendrimer partition function is defined by

$$
q_{\mathrm{D}} \equiv \int \mathrm{d} v_{I} \mathrm{e}^{-E^{*}\left(v_{I}\right)}
$$

while the contribution $Q^{\prime}$ is a conformation-weighted configurational average of the inter-dendrimer interactions, given by

$$
Q^{\prime}=\int \mathrm{d}\{I\} \prod_{I=1}^{N_{\mathrm{D}}} P^{0}\left(v_{I}\right) \prod_{J=I+1}^{N_{\mathrm{D}}} \mathrm{e}^{-U_{I, J}^{*}}
$$

where

$$
P^{0}\left(v_{I}\right) \equiv \mathrm{e}^{-E^{*}\left(v_{I}\right) / q_{\mathrm{D}}}
$$

is defined as the intrinsic probability for the dendrimer $I$ to be found in conformation $v_{I}$ in the absence of any interactions with the other dendrimers of the ensemble.

The expression of the partition function in eqn. (4) allows the formal separation of the free energy of the dendrimer ensemble,

$$
F=-k_{\mathrm{B}} T \ln Q
$$


into a purely conformational, single-dendrimer part $f_{\mathrm{D}}=$ $-k T \ln q_{\mathrm{D}}$ and an inter-dendritic part

$$
F^{\prime}=-k_{\mathrm{B}} T \ln Q^{\prime}
$$

according to

$$
F=N_{\mathrm{D}} f_{\mathrm{D}}+F^{\prime}
$$

The $f_{\mathrm{D}}$ term involves, according to eqn. (5), integration over the conformational states of a single dendrimer. This term however drops out of the phase transition calculations, where only free energy differences are considered, and therefore $F^{\prime}$ is the relevant free energy part for these calculations.

Eqn. (3) and the related expressions in eqn. (4)-(10) define the exact statistical mechanics of the dendrimer ensemble, within the pair-wise additive assumption of the interactions. This assumption already constitutes an approximation on the molecular interactions. For example, it precludes the explicit treatment of molecular polarisability, whose role in liquid crystalline self-organization is not generally insignificant. Within the pair-wise additive limitation, the effects of such many-body interactions can at best be included indirectly, through effective pair potentials. In any case, eqn. (3) is only of formal significance since the integration over the configurational phase space $\{I\}$ cannot be carried out in closed form for any physically relevant form of the interactions. However, this equation and its equivalent forms are useful as a starting point for the systematic development of approximations. Two schemes of such approximations are presented in sections III and IV and are illustrated by applications to specific molecular models.

\section{Interconverting shape approach}

If we assume that the conformational states of the dendrimer are discrete, i.e. that the conformational variable $v_{I}$ assumes discrete values, then the formal integration in eqn. (3) will entail for each dendrimer in the system a summation over all conformations, $\sum_{v_{I}}$, and an integration, $\int \mathrm{d} \varpi_{I}$, over its position and orientation variables denoted collectively by $\varpi_{I}=\left(\boldsymbol{R}_{I} ; \Omega_{I}\right)$.

Suppose that the conformational states can be grouped into sets, with the states in each set exhibiting identical dendrimerdendrimer interaction $U_{I, J}$. For example, in the special case where these interactions are assumed to be hard body repulsions, the grouping would be such that all the members of a set exhibit identical shapes for the dendrimer. ${ }^{23}$ Thus, for brevity we will refer to these sets of conformations as "shapes", although the formulation is applicable to soft potentials as well. The different shapes of dendrimer $I$ are denoted by $S_{I}$ and the distinct conformations associated with the same shape $S_{I}$ are denoted collectively by $v\left(S_{I}\right)$. Then the conformational sum involving dendrimer $I$ in eqn. (6) can be carried out first over all the conformations belonging to a given shape and then over all the different shapes, i.e.

$$
\begin{aligned}
& \sum_{v_{I}} P^{0}\left(v_{I}\right) \mathrm{e}^{-U_{I, J}^{*}}=\sum_{S_{I}}\left[\sum_{v\left(S_{I}\right)} P^{0}\left(v_{I}\right)\right] \mathrm{e}^{-U_{I, J}^{*}}= \\
& \sum_{S_{I}} P_{S_{I}}^{0} G_{S_{I}, S_{J}}\left(\varpi_{I, J}\right)
\end{aligned}
$$

where the intrinsic probability $P_{S I}^{0}$ I of the shape $S_{I}$ is given by the sum of the intrinsic probabilities of all the conformations that correspond to that shape, i.e.

$$
P_{S_{I}}^{0} \equiv \sum_{v\left(S_{I}\right)} P^{0}\left(v_{I}\right)
$$

and

$$
G_{S_{I}, S_{J}}\left(\varpi_{I, J}\right) \equiv e^{-U_{I, J}^{*}}
$$

describes the interactions between any conformation of shape $S_{I}$ with any conformation of shape $S_{J}$. Here $\varpi_{I J}$ denotes the relative positional and orientational variables $\boldsymbol{R}_{I, J}, \Omega_{I, J}$ of the dendrimer pair $I, J$.

With this grouping, eqn. (6) can be put in the equivalent form

$$
Q^{\prime}=\sum_{\left\{S_{I}\right\}} \int \mathrm{d}\left\{\varpi_{I}\right\} \prod_{I=1}^{N_{\mathrm{D}}} P_{S_{I}}^{0} \prod_{J=I+1}^{N_{\mathrm{D}}} G_{S_{I}, S_{J}}\left(\varpi_{I, J}\right)
$$

The configurational free energy of the system, eqn. (9), is approximated according to the variational cluster method ${ }^{26}$ by introducing a variational weight function $\zeta_{S}(\varpi)$ for each set $S$ of conformations and retaining up to two-particle terms in the cumulant expansion. This leads to the following approximate expression for the free energy:

$$
-F^{\prime} / N_{\mathrm{D}} k_{\mathrm{B}} T \approx \ln \bar{\zeta}+\frac{1}{2}\left(N_{\mathrm{D}}-1\right) \ln \langle G\rangle
$$

where $\bar{\zeta}=\sum_{S} P_{S}^{0} \zeta_{S}$, with $\zeta_{S}=\left(1 / 8 \pi^{2} V\right) \int \mathrm{d} \varpi \zeta_{S}(\varpi)$ and the angular brackets denote averaging with respect to the probability distribution

$$
\rho_{S}(\varpi)=P_{S}^{0} \zeta_{S}(\varpi) / 8 \pi^{2} V \bar{\zeta}
$$

namely,

$$
\langle G\rangle \equiv \sum_{S_{I}, S_{J}} \int \mathrm{d} \varpi_{I} \mathrm{~d} \varpi_{J} \rho_{S_{I}}\left(\varpi_{I}\right) \rho_{S_{J}}\left(\varpi_{J}\right) G_{S_{I}, S_{J}}\left(\varpi_{I, J}\right)
$$

The variational weight functions are determined selfconsistently from the conditions

$$
\zeta_{S}(\varpi)=\exp \left[\frac{\left\langle G_{S_{I}}\left(\varpi_{I}\right)\right\rangle-\langle G\rangle}{\langle G\rangle}\right]
$$

with $\left\langle G_{S_{I}}\left(\varpi_{I}\right)\right\rangle=\sum_{S_{J}} \int \mathrm{d} \varpi_{J} \rho_{S_{J}}\left(\varpi_{J}\right) G_{S_{I}, S_{J}}\left(\varpi_{I, J}\right)$.

The form of the dependence of the variational weight function $\zeta_{S}(\varpi)$ on the positional and orientational variables $\varpi_{I}=\left(\boldsymbol{R}_{I} ; \Omega_{I}\right)$ reflects the symmetry of the phase. In our study of the mesomorphic behaviour of dendrimers we consider the following fluid phases:

- isotropic phases, for which $\zeta_{S}(\varpi)$ is independent of position and orientation, $\left.\zeta_{S}(\varpi)\right|_{\text {Iso }}=\zeta_{S}$,

- nematic phases, for which $\zeta_{S}(\varpi)$ is independent of position, $\left.\zeta_{S}(\varpi)\right|_{\mathrm{Nem}}=\zeta_{S}(\Omega)$

- smectic phases, for which $\zeta_{S}(\varpi)$ is independent of the positional coordinates $X, Y$ in the plane of the smectic layers, $\left.\zeta_{S}(\varpi)\right|_{S m}=\zeta_{S}(Z ; \Omega), Z$ being the positional coordinate along the layer normal, 
- columnar phases, for which $\zeta_{S}(\varpi)$ is independent of the positional coordinate $Z$ along the columnar axis, $\left.\zeta_{S}(\varpi)\right|_{\mathrm{Col}}=$ $\zeta_{S}(X, Y ; \Omega)$, where the coordinates $X, Y$ define the plane normal to the columnar axis of the phase.

For the interconverting shape approach ${ }^{22-24}$ to be usable in practice it is necessary that the number of the relevant shapes be not too large, so that a reasonable number of intrinsic probabilities $P_{S}^{0}$ and interactions $G_{S_{I}, S_{J}}\left(\varpi_{I, J}\right)$ be required as input to the calculation. These quantities can be furnished to some coarse grained representation by considering first the conformation statistics and segmental interactions of a single dendrimer in isolation. Having accomplished this, the basic computational step is then the solution of the self-consistency equations, following which the probability distribution of eqn. (16) and the corresponding free energy of eqn. (15) are obtained. The pair distribution function in this approximation is given by $\rho_{S_{I}, S_{J}}{ }^{(2)}\left(\varpi_{I}, \varpi_{J}\right)=\rho_{S_{I}}\left(\varpi_{I}\right) \rho_{\mathrm{S}_{\mathrm{J}}}\left(\varpi_{J}\right) G_{S_{I}, S_{J}}\left(\varpi_{I, J}\right) /<G>$.

The important conformation sets, or shapes, to keep in eqn. (16) are not necessarily the ones that have the highest intrinsic probabilities $P_{S}^{0}$ for an isolated dendrimer but rather the ones that have significant probabilities to occur in the bulk phase of the interacting dendrimers. The probability of finding, in the bulk phase, any conformational state of shape $S$ is given by $P_{S}=P_{S}^{0} \zeta_{S} / \bar{\zeta}$, from which it is seen that for a shape to have significant probability in the bulk phase it is necessary that, aside from its intrinsic probability $P_{S}^{0}$, it should interact with its environment favourably enough to acquire a large value of its self-consistent weight function integral $\zeta_{S}$.

\section{An example: lattice calculations for an interconverting rod-plate model of dendromesogens}

The interconverting shape formulation will be applied here to a very primitive model: the dendrimer is assumed to exhibit just two sets of conformations, i.e. two "shapes", a rod-like and a disc-like, for which the shape indices $S=\mathrm{r}$ and $S=\mathrm{d}$ will be used. In accordance with this crude molecular picture, the interactions are modelled as purely repulsive, with their strength being determined by the extent of overlap between the molecular volumes. Molecular partitioning, and the associated microsegregation of the self-organisation, is introduced into the model by differentiating between the strength of the repulsive interactions among different parts of the rod-like or disc-like objects. In keeping with the simplicity of this modelling, the calculations of the free energy and the selfconsistent weight functions of eqn. (15) and (18) are performed on a cubic lattice. Thus, we assume that the molecules are made up of cubic blocks and are constrained to translate and rotate on a cubic lattice space. The lattice unit cell dimensions are taken to coincide with the size of the molecular building blocks. By restricting the molecules to move so that each of their building blocks occupies a single unit cell of the lattice, the computational effort is reduced considerably, compared to a continuous sampling of the molecular positions and orientations, without severely affecting the predictive aspects of the model.

The same building blocks, in different configurations, are used to construct both the rod and the disc shapes of the dendrimers. The blocks of dendrimer $I$ are enumerated by the
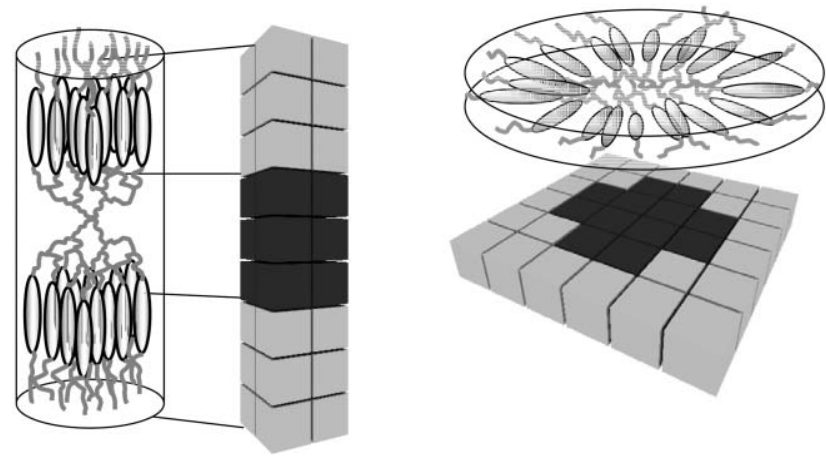

Fig. 1 Schematic representation of the two dominant shapes (rodand disc-like) of a globular dendrimer model of the PAMAM type peripherally functionalized with mesogenic units. Shown are also the respective idealized block structures used in the lattice calculations.

index $b_{I}$. As shown in Fig. 1, two types of blocks are introduced in order to differentiate between chemically distinct parts of the dendrimer. The differentiation is between regions with high density of mesogenic units (light grey blocks) and regions occupied by the flexible chain segments forming the dendritic scaffold (dark grey blocks).

The molecular interactions used for the present lattice calculations are modeled in a pair-wise additive scheme such that the potential between a pair of blocks belonging to different dendrimers vanishes except when these blocks occupy the same or adjacent lattice sites. Accordingly the interaction potential between molecules $I$ and $J$ can be written as

$$
U_{I, J}=\sum_{b_{I}, b_{J}}\left(u_{b_{I}, b_{J}}^{(0)} \delta\left(R_{b_{I}, b_{J}}\right)+u_{b_{I}, b_{J}}^{(1)} \delta\left(\left|R_{b_{I}, b_{J}}\right|-1\right)\right)
$$

where $R_{b_{I}, b_{J}}$ denotes the distance between blocks $b_{I}$ and $b_{J}$, and $u_{b_{I}, b_{J}}^{(0)}, u_{b_{I}, b_{J}}^{(1)}$ stand for the values of the potential for that pair of blocks occupying respectively the same lattice site or adjacent lattice sites. The summation runs over all the blocks of molecules $I$ and $J$.

To study how molecular partitioning influences the phase behaviour of these systems we have considered two different models of the block-block interactions. The first is a purely hard-body model: two blocks, of any kind, are forbidden to occupy the same lattice site and have otherwise no interaction. Obviously, this model disregards any distinction between submolecular parts. The second model is segment-differentiating, using soft and hard repulsions where the following differences are introduced between the flexible scaffold blocks and the mesogenic blocks:

(i) a scaffold block cannot occupy the same lattice site with another block, of any kind, has no interaction with adjacent scaffold blocks and exerts a soft repulsion on any mesogenic block occupying an adjacent site, and

(ii) a mesogenic block exerts soft repulsions, of different intensity, on other mesogenic blocks occupying the same or adjacent lattice sites.

Accordingly, the interaction terms in eqn. (19) for this segment-differentiating model are as follows:

$u_{b_{I}, b_{J}}^{(0)}=\infty$ (hard body) if at least one of the blocks $b_{I}, b_{J}$ is a scaffold lock. 
$u_{b_{I}, b_{J}}^{(0)}$ has a finite positive value (soft repulsion), call it $u$, if both $b_{I}, b_{J}$ are mesogenic locks.

$u_{b_{I}, b_{J}}^{(1)}=0$ (no interaction) if both $b_{I}, b_{J}$ are scaffold locks.

$u_{b_{I}, b_{J}}^{(1)}$ has a finite positive value (soft repulsion), call it $u^{\prime}$, if one of $b_{I}, b_{J}$ is a scaffold block and the other is a mesogenic block.

$u_{b_{I}, b_{J}}^{(1)}$ has a finite positive value (soft repulsion), call it $u^{\prime \prime}$, if both $b_{I}, b_{J}$ are mesogenic locks.

To avoid introducing several independent parameters for the soft repulsion potentials we have used in our calculations a single parameter by setting $u^{\prime}=u / 2$ and $u^{\prime \prime}=u / 4$. The single strength parameter $u$ was then given a representative value by setting $u / k_{\mathrm{B}} T=0.1$.

Having specified the intermolecular interactions, there is just one more input parameter that is required in order proceed with the calculation of the phase diagrams for the interconverting rod-disc model of the dendromesogens. This parameter is the intrinsic probability for one of the two shapes. We have chosen to describe the phase behaviour as a function of the disc intrinsic probability $P_{d}^{0}$. Finally, it should be noted that the dimensions of the rod and disc shapes are fixed according to their block composition shown in Fig. 1. In particular, the two shapes and their respective submolecular parts (mesogenic or scaffold) are of equal volumes since they consist of the same number of blocks of either kind.

The essential step in the calculations ${ }^{22-24}$ consists in solving the self-consistency conditions of eqn. (18). We have calculated phase equilibrium, based on the free energy of eqn. (15), for isotropic, nematic, orthogonal smectic and rectangular orthogonal columnar phases. The calculation is initiated by giving a specific value for the intrinsic probability of the disc-like molecular shape $P_{\mathrm{d}}^{0}$. Then, the dimensionless pressure $p^{*}=$ $p V_{\text {mol }} / k_{\mathrm{B}} T$ (here $p$ is the pressure and $V_{\text {mol }}$ the molecular volume) at which a phase transition occurs is located by solving the coexistence conditions for the two phases. The resulting phase diagrams for the two parameterizations of the model interaction are presented in Fig. 2(a),(b). In both cases the intrinsic probability of the disc-like molecular shape, $P_{\mathrm{d}}^{0}$, is varied in the range from 0 (purely rod-like) to 1 (purely disc-like).

Although the phase diagrams of Fig. 2(a),(b) contain all the essential information for the phase stability of the system, it is experimentally more relevant to introduce the temperature as the thermodynamic variable instead of the intrinsic probability of the molecular shapes. To do that we assume that the intrinsic probabilities for the rod-like (disc-like) shapes may be written as

$$
P_{\mathrm{r}(\mathrm{d})}^{0}=\frac{\exp \left[-\varepsilon_{\mathrm{r}(\mathrm{d})} / k_{\mathrm{B}} T\right]}{\exp \left[-\varepsilon_{\mathrm{r}} / k_{\mathrm{B}} T\right]+\exp \left[-\varepsilon_{\mathrm{d}} / k_{\mathrm{B}} T\right]}
$$

with $\varepsilon_{\mathrm{r}(\mathrm{d})}$ representing effective free energies for the two shapes. On further assuming that the free energy difference $\Delta \varepsilon \equiv \varepsilon_{\mathrm{r}}-\varepsilon_{\mathrm{d}}$ does not vary with temperature appreciably (although the individual free energies $\varepsilon_{\mathrm{r}}$ and $\varepsilon_{\mathrm{d}}$ may do so), we obtain for the scaled reciprocal temperature $\left(\varepsilon_{\mathrm{r}}-\varepsilon_{\mathrm{d}}\right) / k_{\mathrm{B}} T=\ln \left(P_{\mathrm{d}}^{0} /\left(1-P_{\mathrm{d}}^{0}\right)\right)$. Based on this expression, the $\left(p^{*}, P_{\mathrm{d}}^{0}\right)$ phase diagram of Fig. 2(a), (b) are readily transformed into the pressureinverse temperature phase diagrams shown in Fig. 3(a)-(d), with scaled axes $\left(p V_{\mathrm{mol}} /|\Delta \varepsilon|\right)$ and $\left(|\Delta \varepsilon| / k_{\mathrm{B}} T\right)$ respectively.
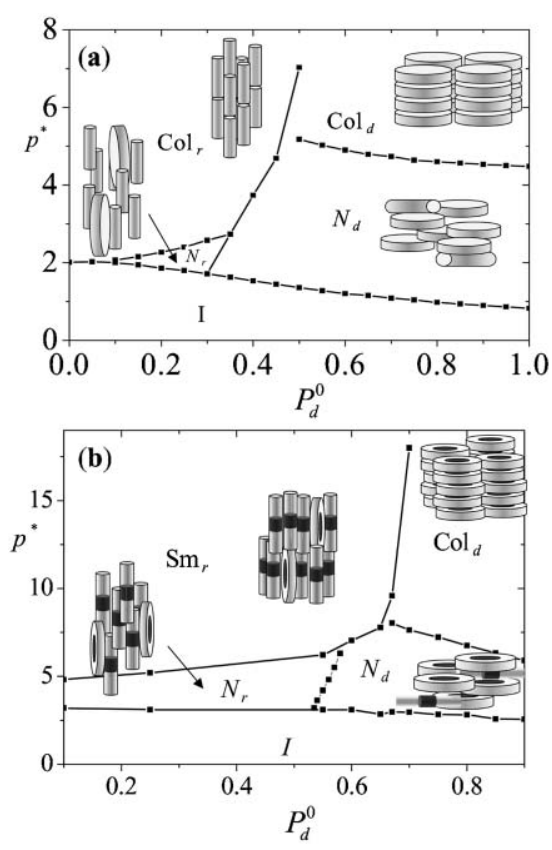

Fig. 2 Calculated $\left(p^{*}, P_{\mathrm{d}}^{0}\right)$ phase diagrams (dimensionless pressure $v s$. intrinsic probability of the disc-like shape) with the interconverting rod-disc model of the dendromesogens for the two parameterizations of the block-block interactions: (a) purely hard-body repulsions between the blocks and (b) interactions differentiating between mesogenic and scaffold blocks. Cartoons of the self-organisation motifs are inserted for each phase on the diagrams. The number of rods and discs included in each cartoon is intended to give a qualitative indication of the relative populations of the two shapes.

As evident from the phase diagrams in Fig. 3(a),(b), when the intermolecular interactions do not distinguish between different parts of the molecules, as in the case of the purely hard body repulsions, the ensuing phase diagrams show the usual phase sequences obtained in hard-rod or hard-disc molecular theories ${ }^{24,27}$ and simulations. ${ }^{28,29}$ In particular, when the rod-like shape is intrinsically more probable than the disc-like $\left(\varepsilon_{\mathrm{r}}<\varepsilon_{\mathrm{d}}\right)$, the system transforms on cooling from the isotropic phase to a uniaxial nematic phase and from there to a positionally ordered phase, Fig. 3(a). In the case of the purely hard repulsive system the positionally ordered phase is columnar, formed by rods that are free to slide side-by-side along the columnar axis of the phase. A similar disappearance of smectic phases in favour of the columnar self-organization has been reported ${ }^{30,31}$ for highly oriented systems of hard cylindrical objects. For relatively low pressures, the calamitic nematic phase is suppressed and the only phase transition is from the isotropic to the columnar phase at rather low temperatures. On the other hand, when the disc-like shape is the one with the larger intrinsic probability $\left(\varepsilon_{\mathrm{d}}<\varepsilon_{\mathrm{r}}\right)$, the phase sequence, at all the pressures, goes, on cooling, from the isotropic to a discotic nematic phase and then on to a columnar phase, Fig. 3(b). Some of the phases, particularly the columnar rod phase, and some of the phase sequences described by these phase diagrams are not observed experimentally for typical liquid crystalline dendrimers. ${ }^{2-9}$

The situation changes dramatically when the submolecular partitioning is incorporated, even in the minimal and perhaps 

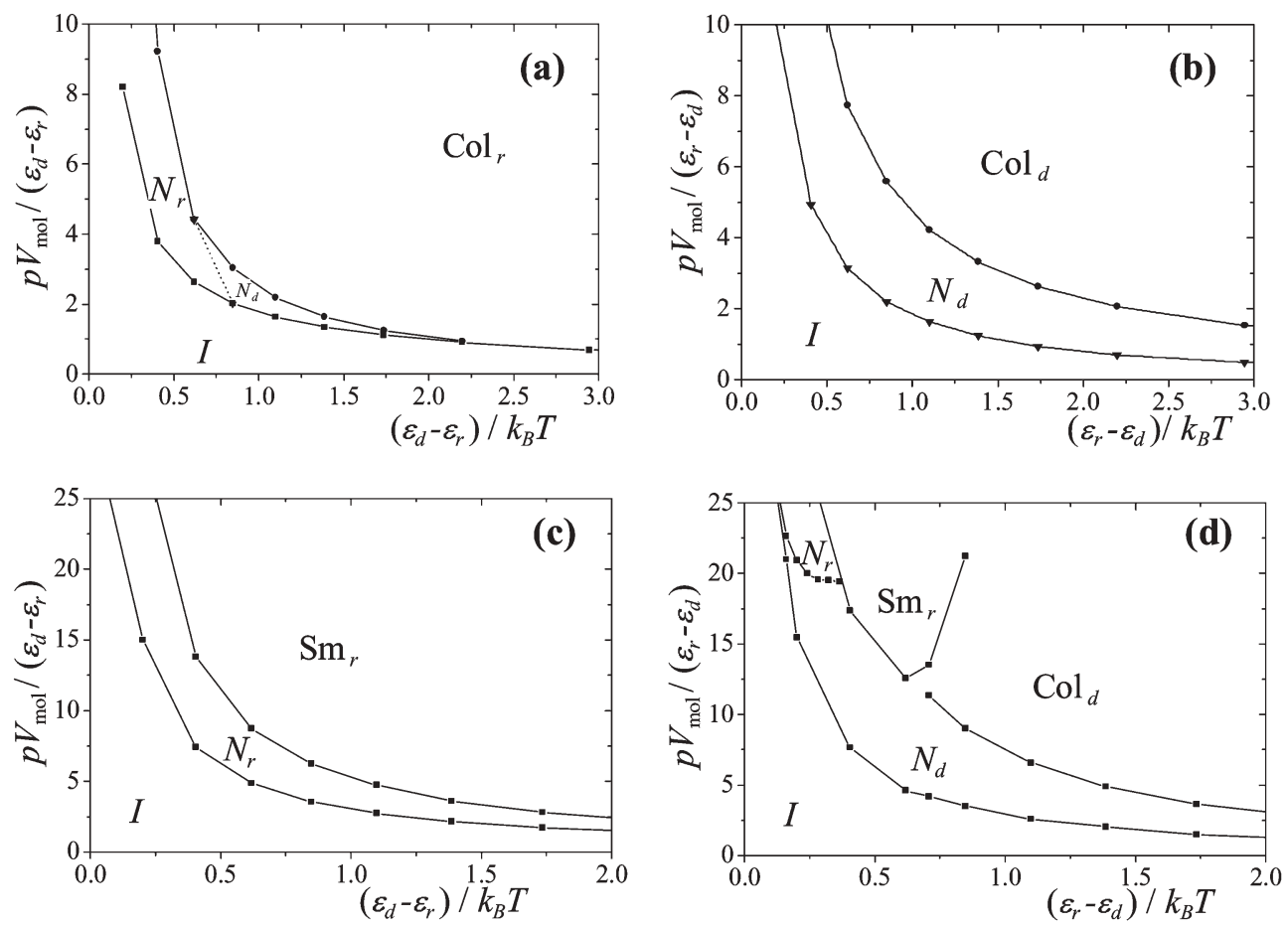

Fig. 3 Calculated phase diagrams (pressure vs. reciprocal temperature) for the interconverting rod-disc model of the dendromesogens for the two parameterizations of the block-block interactions: $(a, b)$ purely hard-body repulsions between the blocks. In (a) the rod shape is taken with lower intrinsic free energy (higher intrinsic probability) than the disc shape while in (b) the rod shape is taken to be the one with the higher free energy; (c,d) the corresponding diagrams for the segment-differentiating parameterization, with (c) corresponding to higher intrinsic abundance of rod shapes and (d) of disc shapes.

oversimplified way it is done in the present calculations. Indeed, on introducing differentiating interactions between the two species of sub-molecular blocks, the variety of phases and of the possible phase sequences becomes richer, as is evident from the diagrams in Fig. 3(c),(d). Thus, when the intrinsically more abundant shape is the disc-like, Fig. 3(d), five phases of different symmetries appear on the pressure vs. temperature phase diagram: the isotropic, two uniaxial nematic phases (one calamitic and one discotic denoted by $N_{\mathrm{r}}$ and $N_{\mathrm{d}}$ respectively), the orthogonal smectic phase (rich in rod-like shapes) and the columnar discotic phase (rich in disc-like shapes). At low pressures the phase sequence is similar to that of the purely repulsive system, i.e. isotropic/discotic nematic/columnar. At moderate pressures, or, equivalently for lower free energy difference of the two molecular shapes, the orthogonal smectic phase is inserted between the discotic nematic phase and the columnar phase. This phase transformation, from lamellar to columnar, is rarely observed in common, low molar mass liquid crystals and, in fact, the few known instances ${ }^{32}$ involve some self-assembly of the entities that self-organise into columns. There are, however, at least two cases of such lamellar-columnar phase transitions reported in the literature. Rueff et al. ${ }^{8}$ and Richardson et al. ${ }^{5}$ in both of which the underlying mechanism is related to the change in the dominant conformation of the dendrimers, in accordance with the results of the present calculations.

When $\varepsilon_{\mathrm{r}}-\varepsilon_{\mathrm{d}} \ll 1$ but still with $\varepsilon_{\mathrm{d}}<\varepsilon_{\mathrm{r}}$, corresponding to nearly equal intrinsic probabilities of the rod-like and the disclike shapes, a phase sequence becomes possible whereby, on cooling from the isotropic (see Fig. 3(d)), a discotic nematic phase is obtained which, on further cooling, is transformed to a rod (calamitic) nematic which in turn cools to an orthogonal smectic phase. This result shows that the intrinsically more abundant shape is not necessarily the dominant one in the ordered bulk phase. A quantitative demonstration of this observation is given in Fig. 4, where we have plotted the calculated bulk probabilities $P_{\mathrm{r}}$ and $P_{\mathrm{d}}$ of the two shapes as function of pressure, at scaled temperature $\left(\varepsilon_{\mathrm{r}}-\varepsilon_{\mathrm{d}}\right) / k_{\mathrm{B}} T=0.71$.

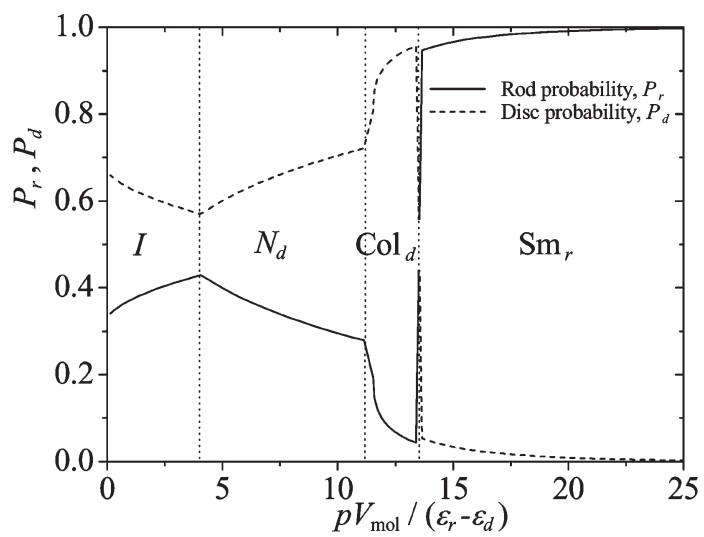

Fig. 4 Calculated bulk probability of the $\operatorname{rod}\left(P_{\mathrm{r}}\right)$ and disc $\left(P_{\mathrm{d}}\right)$ shapes, as a function of the scaled pressure, corresponding to the phase sequence obtained from the diagram in Fig. 3(d) by fixing the value of the scaled temperature at $\left(\varepsilon_{\mathrm{r}}-\varepsilon_{\mathrm{d}}\right) / k_{\mathrm{B}} T=0.71$. 
The plots indicate that all the phase transitions are accompanied by significant chances in the bulk probabilities of the two shapes. Notably, the transition from smectic to columnar is accompanied by a clear and abrupt inversion of the shape populations.

From the results obtained with the segment-differentiating model it can be seen that by employing just two basic shapes and very simple forms for their interactions it is possible to reproduce a rich variety of phase transitions and the associated structural and conformational changes. Lattice calculations using the interconverting shape model have also been successfully applied to the description of systems showing a richer structure of submolecular partitioning, such as fullerene containing liquid crystalline dendrimers. ${ }^{33}$ In this sense, the interconverting shape approach can provide a rationalization of the mesomorphism exhibited by certain classes of dendromesogens ${ }^{2-10}$ in terms of molecular features, such as the global shape and the partitioning into chemically distinct submolecular regions, that can essentially be related directly to the molecular structure. The one ingredient of this approach that is generally more elaborate to deduce from the molecular structure is the intrinsic probability distribution among the dominant shapes. Such deduction would strictly require the input from molecular mechanics calculations on a single dendrimer in order to determine the total probability of the molecular conformations that can be enclosed within a given geometrical shape. However, there are instances where it becomes apparent how one could chemically control the intrinsic probabilities of the basic shapes. For example, it is shown in the work of Rueff et al. ${ }^{8}$ that, by varying the proportion of single to double end chains attached to the mesogenic units of the same dendrimer, and thus modifying the packing mode of these chains within the dendrimer, it is possible to systematically shift the self-organisation trends between rod-like and disc-like dominance. Within the context of the interconverting shape model presented here, the change in the proportion of single to double end chains provides one way of chemically varying the relative intrinsic probabilities of the disc and rod shapes.

By limiting the model calculations to just two shapes, a rod and a disc, both of which possess an axis of full rotational symmetry, it is not possible to describe biaxial or tilted phases. This is, however, not an inherent difficulty of the interconverting shape approach. Shapes such cylinders of elliptical crosssection, oblique cylinders, etc., can be straightforwardly included among the interconverting shapes and indeed the conformation structure and energetics of the PAMAM-type dendromesogens allow for considerable intrinsic probabilities of such global shapes. Similarly, the approach can accommodate polar and chiral shapes. The inclusion of asymmetric shapes complicates somewhat the technical aspects of the computational implementation of the model, starting with the decomposition of the shapes into blocks. In the present illustration of the model we have chosen, for the sake of simplicity, to avoid such complexities by restricting it to just two shapes of full rotational symmetry. This is done at the expense of the richness of the calculated phase diagrams since the occurrence of tilted phases, which are quite common for PAMAM type dendromesogens, ${ }^{7,8}$ is forbidden by the assumed uniaxial symmetry of the self-organising entities. For this reason, a full comparison of the calculated phase diagrams with experimental ones is not warranted at the present stage, even on a qualitative level.

A basic limitation of the interconverting shape approach is that it becomes inadequate for the description of phenomena where the internal motions of the dendrimer are directly involved. Thus, for example, the static dielectric properties can be described by assigning to each shape a molecular polarisability tensor and a molecular dipole moment (usually vanishing by symmetry) and considering reorientations and translations of the dendrimer as a whole. This constitutes, of course, a severely oversimplified molecular picture of the dielectric behaviour of the actual systems, except perhaps for extreme cases of internally very dense dendrimers precluding any significant intra-dendrimer segmental rearrangements. ${ }^{23}$ The picture can be improved by considering distributed polarisabilities and dipole moments over the dendrimer volume and by further allowing for some deformation modes of the basic shapes. This however makes the approach more complex and requires a larger set of input information to be furnished with the guidance of the atomistic structure and conformational statistics of the single dendrimer. Similarly, the description of the dynamic behaviour of the dendrimers with this approach is meaningful only for time scales pertaining to global motions of the dendrimer shapes and of their basic deformation modes while it is clearly inapplicable for time scales associated with intra-dendrimer segmental rearrangements. ${ }^{34}$

\section{The constrained segment approach}

Rather than viewing the entire dendrimer as the basic molecular unit, this description focuses on the sub-dendritic units that give rise to the mesomorphic behaviour of the dendrimer ensemble. Denoting the segmental interaction Boltzmann factors by $G\left(i_{I_{2}} j_{J}\right)=\exp \left(-u\left(i_{I}, j_{J}\right) / k_{\mathrm{B}} T\right)$, we may rewrite eqn. (3), taking into account eqn. (1) and (2), as follows

$$
Q=\int \mathrm{d}\{I\} \prod_{I=1}^{N_{\mathrm{D}}} \prod_{i_{I} \neq j_{I}} G\left(i_{I}, j_{I}\right) \prod_{J=I+1}^{N_{\mathrm{D}}} \prod_{i_{I}^{\prime}, j_{J}^{\prime}} G\left(i_{I}^{\prime}, j_{J}^{\prime}\right)
$$

in which the interaction terms associated with intra-dendrimer pairs of segments are explicitly separated from those of the inter-dendrimer pairs. In a more compact notation, all the segments in the ensemble are labeled by a single index $i, j \ldots=1,2,3 \ldots N$, without specific reference to the dendrimer they belong to. Here $N=N_{\mathrm{S}} N_{\mathrm{D}}$ is the total number of segments in the ensemble. The partition function expression assumes then the form

$$
Q=\int \mathrm{d}\{I\} \prod_{i \neq j} G(i, j)
$$

with the understanding that the different pairs $i, j$ are not necessarily equivalent.

The variational cluster method ${ }^{26}$ can then be applied to eqn. (21) to obtain the free energy of the system up to twosegment terms in the cumulant expansion. Denoting the variational weight function introduced for each segment $i$ 
by $\zeta(i)$, we have for the probability distribution of a single segment

$$
\rho(i)=\zeta(i) / \zeta_{i}
$$

with $\zeta_{i} \equiv \int \mathrm{d}(i) \zeta(i)$ denoting the integrated weight function for segment $i$. The probability distribution for a pair of segments is given in this approximation by

$$
\rho^{(2)}(i, j)=\rho(i) \rho(j) G(i, j) /<G_{i, j}>
$$

where

$$
\left\langle G_{i, j}\right\rangle \equiv \int \mathrm{d}(i) \mathrm{d}(j) \rho(i) \rho(j) G(i, j)
$$

and the expression for the approximate free energy is

$$
-F / k_{\mathrm{B}} T \approx \sum_{i} \ln \zeta_{i}+\sum_{i \neq j} \ln \left\langle G_{i, j}\right\rangle
$$

The variational weight functions are determined by functional minimization of the above expression for the free energy which leads to the self-consistency equations

$$
\zeta(i)=\exp \left[\sum_{j} \frac{\left\langle G_{j}(i)\right\rangle-\left\langle G_{i, j}\right\rangle}{\left\langle G_{i, j}\right\rangle}\right]
$$

where

$$
\left\langle G_{j}(i)\right\rangle=\int \mathrm{d}(j) \rho(j) G(i, j)
$$

The dependence of the variational weight function $\zeta(i)$ on the positional and orientational variables reflects the symmetry of the phase under consideration, as detailed in section III, only now these variables refer to the $i^{\text {th }}$ segment rather that the entire dendrimer.

The input information for the calculations with the segmental approach are the interaction potentials for the pairs of inter-dendritic segments as well as those of the intradendritic pairs, the latter differing from the former in that they include the additional energetic contribution associated with their connectivity through the dendritic scaffold. The segmental approach rests on the partitioning of the conformational energy of the dendrimer among the intra-dendritic pairs of segments, as indicated in eqn. (21). This cannot always be done in a unique way. Rather than addressing this issue in a general way, we consider here a specific example of the application of this approach to a globular dendrimer functionalized peripherally with mesogenic units

\section{The dimer ensemble model of dendromesogens}

To present a concrete application of the segmental approach we consider the dendritic architecture shown in Fig. 5. The dendrimer, of the PAMAM type, ${ }^{2}$ has a flexible scaffold of symmetrical radial topology, with three branches radiating at each branching point. The periphery of the dendrimer is functionalised by attaching identical rodlike mesogenic units to the terminal branches. This architecture is representative of a class of extensively studied dendromesogens. ${ }^{2,5-8}$

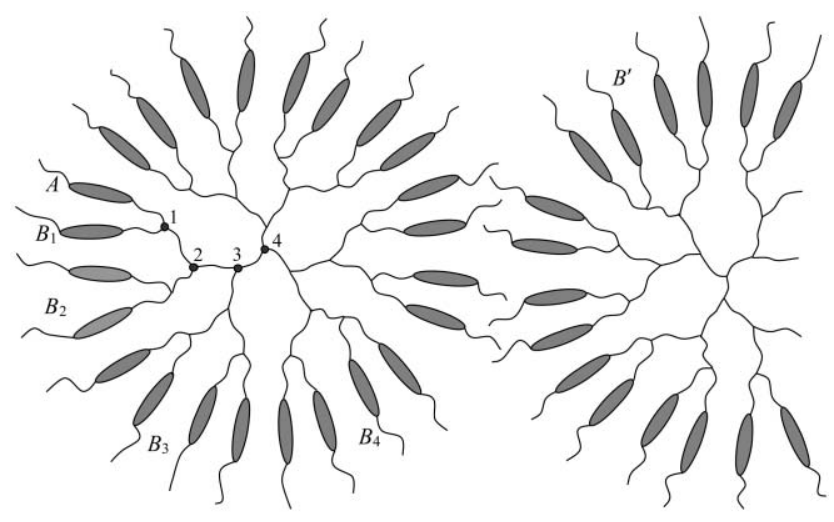

Fig. 5 Fourth generation PAMAM-type dendrimer topology showing the hierarchy of interactions among intra-dendrimer mesogenic pairs $\mathrm{AB}_{\mathrm{b}}$ according to the order of the branching points $b=1,2,3,4$ present in the branch path connecting the members of each type of pair. An inter-dendrimer pair $\mathrm{AB}^{\prime}$ is also indicated.

Two mesogenic units $i, j$ belonging to different dendrimers are said to form a non-bonded pair, such as the pair $\mathrm{AB}^{\prime}$ in Fig. 5, and are assigned an interaction $u_{\mathrm{n}}(i, j)=u_{\mathrm{n}}\left(\boldsymbol{r}_{i j}, \omega_{i j}\right)$ that depends on the relative position $\boldsymbol{r}_{i j}$ and the relative orientation $\omega_{i j}$ of these units. The interaction of bonded pairs, i.e. pairs of mesogenic units belonging to the same dendrimer, consists of the non-bonded interaction and an additional term $u_{\text {path }}$ associated with the conformational constraints imposed on the relative positions and orientations of the two units as a result of their attachment at the ends of a path formed by chain segments (branches) of the scaffold. Accordingly, the bonded potential $u_{\mathrm{b}}$ for a pair $i, j$ is a function of the conformational variables $v_{i, j}$ of the branch-path that connects them,

$$
u_{\mathrm{b}}(i, j)=u_{\mathrm{b}}\left\{v_{i j}\right\}=u_{\mathrm{n}}\left(\boldsymbol{r}_{i, j}\left\{v_{i j}\right\}, \omega_{i, j}\left\{v_{i j}\right\}\right)+u_{\mathrm{path}}\left\{v_{i j}\right\}
$$

For simplicity, the flexible branches of the scaffold are not directly assigned any anisotropic interaction. They are assumed to exist in a number of discrete conformations, generated according to the RIS scheme ${ }^{35}$ with equal intrinsic probabilities; aside from that, they simply fill space in the dendritic interior. Accordingly, while the dendrimer in this model is considered to be built of two kinds of sub-units, namely the elongated mesogenic cores and the various rigid segments that are jointed together to form the flexible scaffold, only the mesogenic cores are endowed with orientability.

Under these assumptions, the pair-wise summation appearing in the free energy expression of eqn. (26) involves a limited number of inequivalent types of mesogenic pairs. Thus, for the specific example of fourth generation dendrimer shown in Fig. 5, there are altogether five distinct types of pair terms $\left\langle G_{i, j}\right\rangle$. One type is formed by mesogenic units belonging to different dendrimers i.e. non-bonded pairs $\mathrm{AB}^{\prime}$ in Fig. 5. With the $N_{\mathrm{S}}=24$ mesogenic units of each dendrimer being identical, and equivalent by virtue of their symmetric attachment on the dendritic periphery, there are altogether $N N_{\mathrm{S}}\left(N_{\mathrm{D}}-1\right) / 2$ equivalent non-bonded pair terms $\left\langle G_{i, j}\right\rangle \equiv\left\langle G_{\mathrm{n}}\right\rangle$ in an ensemble of $N_{\mathrm{D}}$ dimers (which therefore contains in total $N=N_{\mathrm{S}} N_{\mathrm{D}}$ mesogenic units). 
The other four types of pair terms $\left\langle G_{i, j}>\right.$ are intra-dendritic, formed, as shown in Fig. 5, by first $\left(\mathrm{AB}_{1}\right)$, second $\left(\mathrm{AB}_{2}\right)$, third $\left(A B_{3}\right)$ and fourth $\left(A_{4}\right)$ neighbours on the same dendrimer. We shall denote these bonded-pair terms by $\left\langle G_{b}\right\rangle$, with the index $b=1,2,3,4$ specifying their neighbour order within the dendrimer. With the number of equivalent pairs of neighbour order $b$ denoted by $N h_{b}$ in the ensemble, we have for the $h_{b}$ factors the values $h_{1}=1 / 2, h_{2}=1, h_{3}=2, h_{4}=8$.

According to the above considerations, and noting that the $N$ mesogenic units in the system, being equivalent, are described by a single integrated weight $\zeta$, the segmental summation in the free energy of eqn. (26) can be carried out to yield the expression

$$
-F / N k_{\mathrm{B}} T=\ln \zeta+\frac{1}{2} N_{\mathrm{S}}\left(N_{\mathrm{D}}-1\right) \ln \left\langle G_{n}\right\rangle+\sum_{b=1}^{4} h_{b} \ln \left\langle G_{b}\right\rangle
$$

The bonded contributions of different neighbour orders in this expression correspond to typical mesogenic dimers, i.e. pairs of mesogenic units linked by a flexible spacer. ${ }^{36}$ The spacer in this case is the branch-path that connects the mesogenic pair. Accordingly, the dendrimer system is treated as an ensemble of mesogenic dimers with identical terminal units but different spacers. The proportion of each type of spacer in the dimer ensemble is governed by the factors $h_{b}$. From this stage on, the results obtained within the molecular theory of liquid crystalline dimers ${ }^{37-39}$ can be carried over straightforwardly to the description of the dendrimers, thus providing a way to phase transition calculations, evaluation of segmental order parameters, pair correlation averages and dynamic response on the time scale associated with segmental motions. These calculations start from the interactions of the mesogenic units and the conformation structure of the pertinent branch-paths within the dendritic scaffold. It is known that the mesomorphic behaviour of dimers could be very sensitive to the structure and length of the spacer. ${ }^{36}$ This sensitivity is carried over to the individual bonded-pair terms that are superimposed to form the free energy of eqn. (30).

The free energy expression in eqn. (30) is not restricted to symmetric dendrimers of the type shown in Fig. 5 but can be applied to the description, within the dimer ensemble model, of any system of mesogenic units connected by flexible spacers, including main chain and side chain liquid crystalline oligomers and polymers as well as mesogenic hyper-branched polymers and networks. For example, written in the form

$$
-F / N k_{\mathrm{B}} T=\ln \zeta+\frac{1}{2} \ln \left\langle G_{n}\right\rangle^{N}+\sum_{b} h_{b} \ln \left\langle G_{b}\right\rangle
$$

the free energy of eqn. (30) can be applied to the entire hierarchy of linearly linked mesogenic units shown in Fig. 6a. With $N$ mesogenic units in the system, the purely monomeric ensemble is described by setting $h_{b}=0$ in eqn. (31) for any $b$ (all the pairs in the ensemble are non-bonded). The dimer ensemble is obtained by setting $h_{1}=1 / 2$ and $h_{b}=0$ for $b>1$ (only non-bonded and first neighbour bonded pairs exist in the ensemble). It readily follows that for an ensemble of linear symmetric oligomers of order $N_{0}$ (with $N_{0}$ denoting the number of mesogenic units in the oligomer) the factors $h_{b}$ are given by $h_{b}=\left(N_{0}-b-1\right) / N_{0}$ for $b=1, \ldots, N_{0}-1$ and $h_{b}=0$ for $b \geqslant N_{0}$.

The fact that, for large $N_{0}$, high rank neighbour terms are present in eqn. (31) does not in general preclude the applicability of this approach to large linear oligomers and main chain polymers or to high generation dendrimers. Thus, if the spacers are sufficiently long and flexible the orientational correlations among distant neighbours become weak and the respective $\ln \left\langle G_{b}\right\rangle$ terms can be ignored when using eqn. (31) to describe the nematic to isotropic phase transition. Calculations using standard rotational potentials to generate the conformations of alkyl spacers show that the orientational correlations between bonded mesogenic units become negligibly weak as the length of the spacer (measured by the number of carbon atoms) increased above a dozen carbons. ${ }^{37}$ Accordingly, with spacers of 6 carbons or longer, it is possible to ignore the contributions of second (and more distant) neighbours in eqn. (31). Calculations for the nematic-isotopic phase transition using eqn. (31) with only first neighbour bonded terms have been performed for the linear oligomer systems and for the null generation dendromesogen (star trimer) shown in Fig. 6, for spacer lengths of six carbons or longer.

In these calculations only hard-body interactions were considered among the mesogenic units, which were taken to be hard spherocylinders ${ }^{24}$ of aspect ratio $L / D=4$. The diameter $D$ of the spherocylinder was taken to be 4 times the length of a carbon-carbon bond of the alkyl spacer. The conformations of the spacers were generated according to the standard 3-state

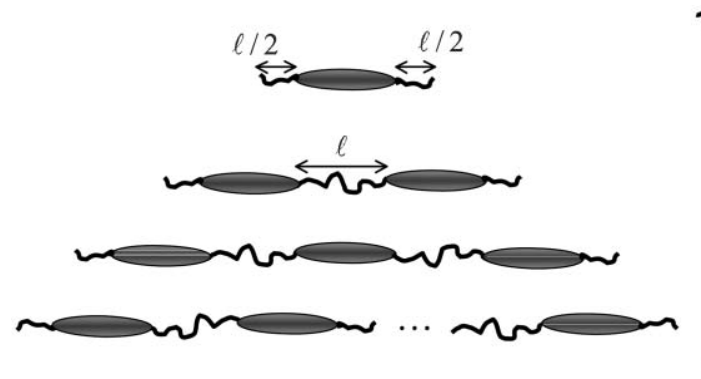

(a)

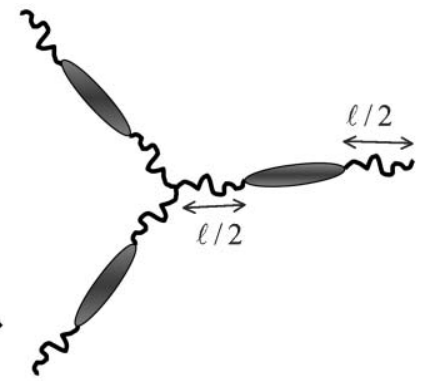

(b)

Fig. 6 (a) Hierarchy of linearly linked repeat units consisting of a rod-like mesogenic core with flexible chains attached at each end. Monomer, dimer, trimer and polymer members of the hierarchy are shown. The length of the spacer is denoted by $\ell$. (b) The same repeat units are linked to form a null generation dendrimer (symmetric star trimer). 
RIS model, ${ }^{35}$ rejecting self-intersecting conformations and assuming for simplicity that the trans state and the gauche \pm states are energetically equivalent. The torsional minima are located at $0^{\circ}$ for the trans state of a $\mathrm{C}-\mathrm{C}$ bond and at $\pm 112^{\circ}$ for the gauche states. Librations of $\pm 7^{\circ}$ about the three torsional minima are allowed in order to dismiss excessive artificial correlations between the two ends of the spacer. Due to this simplified conformation generation scheme and the purely hard body interactions used, the system becomes athermal (the temperature does not enter explicitly in the calculations) and the phase transition can be obtained only by varying the density. Qualitative comparisons can be made, however, with experimental trends of thermotropic systems by associating the densities at the transition with inverse temperatures.

The results on the phase transition densities for the linear dimer, the trimer, the polymer and the null generation symmetric dendrimer (star trimer) of Fig. 6 are shown in Fig. 7 as a function of the spacer length. For the star trimer there are only first neighbor pairs (3 such pairs per trimer). Accordingly the respective factors in eqn. (31) are $h_{1}=1$ and $h_{b}=0$ for $b>1$. Consequently, if more distant than first neighbour pairs are ignored, the free energy for the star trimer is identical to that of the polymer (for which $h_{1}=1$ but $h_{b} \neq 0$ for $b>1)$. The conformations of the spacers in these two systems are of course different as a result of geometrical differences between the central carbon-carbon bonds of the polymer spacer and the central nitrogen-carbon bonds of the star trimer. If such differences are disregarded, as was done for simplicity in the present calculations, the numerical results for the transition densities of the polymer and the star trimer will coincide. It should be noted, however, that the spacer path linking any two monomers of a symmetric star trimer always has an odd number of chain segments whereas for the polymer, and the other linear oligomers, the spacer can have both even and odd numbers of chain segments.

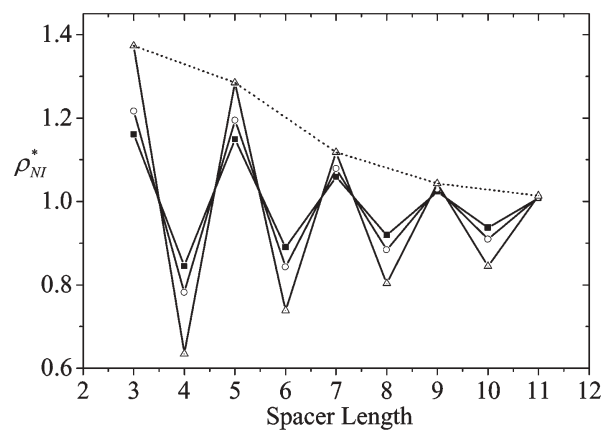

Fig. 7 Calculated dependence of the nematic-isotropic phase transition density $\rho_{N I}^{*}$ on the length of the alkyl spacer for the linearly linked dimer (rectangles), trimer (circles), polymer (triangeles) and the symmetric star trimer (triangles) shown in Fig. 6. The length is measured by the number of carbons in the alkyl spacer and corresponds to $\ell$ of Fig. 6. The density $\rho_{N I}^{*}$ is expressed in units of the phase transition density for the monomer system shown in Fig. 6 . For the reasons explained in the text, the transition densities of the star-trimer coincide with those of the polymer for odd spacer lengths (the points connected by the dashed line) while even spacer lengths are not applicable to symmetric star trimers.
The results in Fig. 7 indicate that, in spite of the extreme simplicity of the interactions and the conformation model used, the basic experimental trends observed for the systems of linear oligomers and polymers ${ }^{40-42}$ are conveyed. In particular, the odd-even alternations in the transition densities (equivalently inverse temperatures) are readily reproduced, with their amplitude decreasing with increasing spacer length and increasing on going from dimers to trimers and then to polymers. These results are also in qualitative agreement with those of self-consistent molecular field calculations based on considerably more elaborate models for the conformations and the segmental interactions. ${ }^{38}$ Due to the symmetry of the spacers connecting the mesogenic pairs of the star trimers, parity alternations are not exhibited by these systems on changing the length of the three spacer arms. This model predicts, however, that such alternations could be introduced on inserting into the sequence asymmetric star trimers with one spacer arm differing in length from the other two by a single carbon atom. Finally, it is worth noting that the differences in the phase transition behaviour of the various systems in Fig. 7, for the same spacer length, are obtained solely on the basis of the topological changes associated with the modes of linking the same number of repeat units (mesogenic cores and alkyl chains) into different supermolecular entities.

\section{Conclusions}

We used the variational cluster method to approximate, to second order terms in the cumulant expansion, the free energy of an ensemble of dendrimers. We developed two approximation schemes, a global and a segmental one, in order to address different aspects of the molecular theory of liquid crystalline dendrimers.

In the global approach with interconverting shapes, the dendrimers are viewed as the elementary entities in the ensemble. This approach is therefore suitable for the description of the self organisation of the entire dendrimers in terms of their dominant conformations and the respective global dendrimer-dendrimer interactions. These are dictated by a coarse grained parameterisation of the atomistic structure of the dendrimer. Even in its most primitive form (with dendrimers consisting of two chemically distinct components and limited to just two interconverting uniaxial shapes, a rodlike and a disc-like, that are restricted to move on a cubical lattice) the global approach accounts for all the experimentally observed dendromesogen liquid crystalline phases that can be obtained within the uniaxiality restriction on the shapes of the self-organising entities. It also predicts a rich variety of phase sequences depending on the intrinsic populations of the two conformations. In particular, it accounts for the lamellarcolumnar phase transitions and predicts nematic-nematic transitions associated with changes in the dominant conformations of the dendrimer.

In the segmental approach, the basic entities in the ensemble are identified with the mesogenic groups of the dendrimer. The connectivity of these groups within the dendrimer is conveyed by an effective potential that can be derived from the conformational statistics of the dendritic scaffold. With this approach, the ensemble of dendromesogens reduces to an 
ensemble of mesogenic dimers with spacers of different lengths, corresponding to the different branch paths within the dendritic scaffold. The segmental approach is suitable for the description of the mesomorphic properties that are sensitive to the ordering and the motion of dendritic segments rather than of the dendrimer as a whole. However, it should be kept in mind that the dimer picture of the segmental approach is obtained by ignoring correlations of more than two mesogenic segments at a time, and therefore its reliability is restricted to situations not involving strongly correlated collective movements of the dendrimer constituents. The segmental approach, on the other hand, provides a unified description for a broad variety of supermolecular and macromolecular mesogens with different linkage topologies of their repeat units.

\section{Acknowledgements}

Support from the RTN Project "Supermolecular Liquid Crystal Dendrimers - LCDD" (HPRN-CT2000-00016) is acknowledged. AGV acknowledges support through the "Caratheodores" research programme of the University of Patras.

\section{Alexandros G. Vanakaras* and Demetri J. Photinos}

Department of Materials Science, University of Patras, Patras 26504, Greece.E-mail:vanakara@upatras.gr;photinos@upatras.gr

\section{References}

1 U. Stebani, G. Lattermann, M. Wittenberg and J. H. Wendorff, J. Mater. Chem., 1997, 7, 607.

2 J. Barberá, M. Marcos and J. L. Serrano, Chem. Eur. J., 1999, 5, 1834.

3 G. H. Mehl and J. W. Goodby, Chem. Ber., 1996, 129, 521.

4 G. H. Mehl and J. W. Goodby, Angew. Chem., Int. Ed. Engl., 1996, 35, 2641; R. Elsäßer, G. H. Mehl, J. W. Goodby and D. J. Photinos, Chem. Commun., 2000, 851.

5 R. M. Richardson, S. A. Ponomarenko, N. I. Boiko and V. P. Shibaev, Liq. Cryst., 1999, 26, 101; S. A. Ponomarenko, N. I. Boiko, V. P. Shibaev, R. M. Richardson, I. J. Whitehouse, E. A. Rebrov and A. M. Muzafarov, Macromolecules, 2000, 33, 5549 .

6 M. Marcos, R. Giménez, J. L. Serrano, B. Donnio, B. Heinrich and D. Guillon, Chem. Eur. J., 2001, 7, 1006.

7 B. Donnio, J. Barberá, R. Giménez, D. Guillon, M. Marcos and J. L. Serrano, Macromolecules, 2002, 35, 370.

8 J.-M. Rueff, J. Barbera, B. Donnio, D. Guillon, M. Marcos and J.-L. Serrano, Macromolecules, 2003, 36, 8368.

9 V. Percec, P. Chu, G. Ungar and J. Zhou, J. Am. Chem. Soc., 1995, 117, 11441.

10 L. Gehringer, D. Guillon and B. Donnio, Macromolecules, 2003, 36, 5593.

11 V. Percec, W.-D. Cho and G. Ungar, J. Am. Chem. Soc., 2000, 122, 10273 and references therein.
12 A. D. Schluter and J. P. Rabe, Angew. Chem., Int. Ed., 2000, 39, 864.

13 N. Ouali, S. Mery and A. Skoulios, Macromolecules, 2000, 33, 6185.

14 C. Tschierske, J. Mater. Chem., 2001, 11, 2647.

15 F. Ganazzoli and R. La Ferla, J. Chem. Phys., 2000, 113, 9288.

16 F. Ganazzoli, R. La Ferla and G. Terragni, Macromolecules, 2000, 33, 6611.

17 C. N. Likos, M. Schmidt, H. Lowen, M. Ballauff, D. Potschke and P. Lindner, Macromolecules, 2001, 34, 2914.

18 N. Zacharopoulos and L. G. Oikonomou, Macromolecules, 2002, 35, 1814.

19 I. O. Gotze and C. N. Likos, Macromolecules, 2003, 36, 8189.

20 H. M. Harreis, C. N. Likos and M. Ballauff, J. Chem. Phys., 2003, 118, 1979.

21 M. Ballauff and C. N. Likos, Angew. Chem., Int. Ed., 2004, 43, 2998.

22 A. F. Terzis, A. G. Vanakaras and D. J. Photinos, Mol. Cryst. Liq. Cryst., 1999, 330, 517.

23 A. F. Terzis, A. G. Vanakaras and D. J. Photinos, Mol. Cryst. Liq. Cryst., 2000, 352, 265.

24 A. G. Vanakaras and D. J. Photinos, J. Mater. Chem., 2001, 11, 2832.

25 A. R. Khokhlov and A. N. Semenov, Physica A, 1982, 112, 605; A. Ten Bosch, P. Maissa and P. Sixou, J. Chem. Phys., 1983, 79, 3462; M. Warner, J. M. F. Gunn and A. B. Baumgartner, J. Phys. A, 1985, 18, 3007; X. J. Wang and M. Warner, Liq. Cryst., 1992, 12, 385.

26 T. Tanaka, Methods of Statistical Physics, Cambridge University Press, Cambridge, UK, 2002.

27 A. G. Vanakaras and D. J. Photinos, Mol. Cryst. Liq. Cryst., 1997, 299, 65.

28 S. C. McGrother, D. C. Williamson and G. Jackson, J. Chem. Phys., 1996, 104, 6755.

29 G. A. C. Veerman and D. Frenkel, Phys. Rev. A, 1992, 45, 5632.

30 A. Stroobants, H. N. W. Lekkerkerker and D. Frenkel, Phys. Rev. A, 1987, 36, 2929.

31 A. M. Somoza and P. Tarazona, Phys. Rev. A, 1989, 40, 4161.

32 S. Diele, S. Grande, J. Kain, G. Pelzl and W. Weissflog, Mol. Cryst. Liq. Cryst., 2001, 362, 111.

33 S. D. Peroukidis, A. G. Vanakaras and D. J. Photinos, J. Mater. Chem., 2005, 15, DOI: 10.1039/b419478k.

34 A. Van-Quynh, D. Filip, C. Cruz, P. J. Sebastiao, A. C. Ribeiro, J. M. Ruef, M. Marcos and J. L. Serrano, J. Mater. Chem., 2005, 15, DOI: $10.1039 / \mathrm{b} 416035 \mathrm{e}$.

35 P. J. Flory, Statistical mechanics of chain molecules, Interscience Publishers, New York, 1969.

36 C. T. Imrie and G. R. Luckhurst, in Handbook of Liquid Crystals Vol. 2B, Low Molecular Weight Liquid Crystals, ed. D. Demus, J. Goodby, G. W Gray, H.-W. Spiess and V. Vill, Wiley-VCH, Weinheim, 1998, ch. X.

37 P. K. Karahaliou, A. G. Vanakaras and D. J. Photinos, Liq. Cryst., in press.

38 H. S. Serpi and D. J. Photinos, J. Chem. Phys., 1996, 105, 1718.

39 H. S. Serpi and D. J. Photinos, Mol. Cryst. Liq Cryst., 2000, 352, 205.

40 C. Ober, J. I. Jin and R. W. Lenz, Polym. J., 1982, 14, 9; A. Roviell and A. Sirigu, Macromol. Chem., 1982, 183, 895; A. Blumstein and O. Thomas, Macromolecules, 1982, 15, 1264.

41 G. R. Luckhurst, Recent advances in Liquid Crystalline Polymers, ed. L. L. Chapoy, Elsevier, Amsterdam, 1984.

42 P. A. Henderson, A. G. Cook and C. T. Imrie, Liq. Cryst., 2004, 31, 1427. 